PESQUIMAT, Revista de la F.C.M. de la

Universidad Nacional Mayor de San Marcos

Vol. XIX N² 2 , pp. 14-25, Julio-Diciembre 2016

\title{
Una Variante del Teorema de la Amistad
}

\section{Montoro E. ${ }^{1} \quad$ Huamán $C .{ }^{1} \quad$ Castillo E. ${ }^{1} \quad$ Melgarejo G. ${ }^{1} \quad$ Armas E. ${ }^{1}$ Sampertegui $M .^{1}$}

(Recibido: 18/04/2016 - Aceptado: 01/07/2016)

Resumen: Con la ayuda de la teoría de grafos y del álgebra lineal se puede dar una demostración a una variante del teorema de la amistad.

Palabras Claves: Teorema de la amistad, Teoría de grafos y álgebra lineal.

\section{A Variant of The Theorem of Friendship}

Abstract: With the help of graph theory and linear algebra can give a demonstration a variant of the theorem of friendship.

Key Words: Theorem of friendship, graph theory and linear algebra.

\section{Introducción}

El Teorema de la Amistad fue dado a conocer por primera vez en 1930 gracias al matemático y filósofo Frank Plumpton Ramsey en un trabajo titulado "On a Problem in Formal Logic" (ver [2]). El Teorema de la Amistad nos dice que:

En cualquier grupo de seis personas, existen tres personas que son mutuamente conocidas o mutuamente desconocidas.

En el presente artículo demostramos la siguiente variante (Ver [1]):

Consideremos una fiesta con $n$ personas, donde cada dos personas tienen exactamente un amigo en común. Entonces alguien conoce a todas las otras personas en la fiesta.

Cabe mencionar que esta variante ya ha sido estudiada y probada por diferentes autores. Nosotros aquí presentamos una demostración diferente.

\footnotetext{
${ }^{1}$ UNMSM, Facultad de Ciencias Matemáticas, e-mail: cdinsonmontoro@yahoo.com
} 


\section{Metodología}

\subsection{Teorema Central}

El resultado principal de este trabajo está dado por el siguiente teorema:

Considérese una fiesta con $n$ personas, donde cada dos personas tienen exactamente un amigo en común. Entonces alguien conoce a todas las otras personas de la fiesta.

\section{Demostración.}

1) La demostración se desarrollará a través de los siguientes pasos:

Paso 1: Enumeramos a las personas de la fiesta de manera conveniente: (1), (2), (3), ..., (n).

Paso 2: Definimos el conjunto $A_{i}$ para cada persona $i$ como el conjunto de amigos de $i$.

Paso 3: $\operatorname{Los} A_{i}, i=1,2,3, \ldots, 2 k+1$ forman una partición del conjunto de las personas de la fiesta, donde cada dos personas tienen exactamente un amigo en común.

Paso 4: Si alguien en la fiesta conoce exactamente a dos personas. Entonces $A_{2}$ ó $A_{3}$ es igual al conjunto vacío y el teorema es válido.

Paso 5: Si alguien conoce a más de dos personas. Entonces: Sean $i, j$ dos amigos de la persona (1) que no se conocen. Entonces $A_{i}$ y $A_{j}$ tienen el mismo número de elementos. Todos los conjuntos $A_{i}, 2 \leq i \leq 2 k+1$, tienen el mismo número de elementos.

Paso 6: Notemos que la elección de la persona que llamamos (1), fue totalmente arbitraria. Luego todas las personas en la fiesta conocen al mismo número de personas.

Paso 7: $n=1+2 k+2 k(2 k-2)$, luego $n=4 k^{2}-2 k+1$ donde la única solución para $k$ es $k=1$.

Luego, la fiesta está compuesta por $n=3$ personas y por lo tanto existe una persona que conoce a todos en la fiesta.

2) Elegimos a cualquier persona como la persona (1). Entonces se tiene por definición

$$
A_{1}=\{\text { Amigos de (1), pero no esta el (1) }\}
$$

Por hipótesis, cada dos personas tienen exactamente un amigo común, por tanto $\left|A_{1}\right|=2 k$ (número par). La arista entre un par de nodos significa la relación de amistad entre ambos nodos.

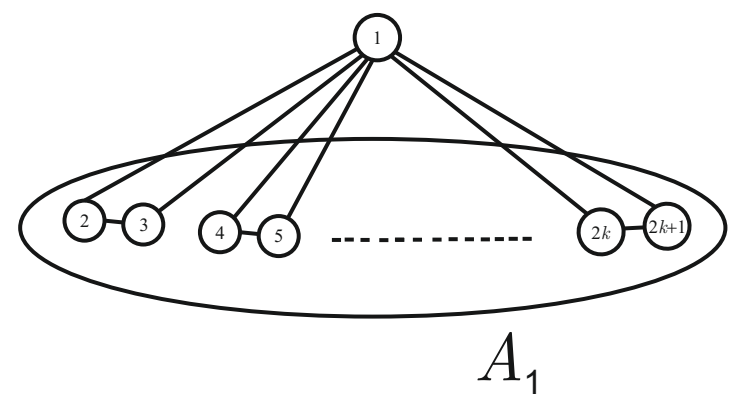


3) Probemos el Paso 3.

Sea $i \neq j \neq 1$ entonces $A_{i} \cap A_{j}=\varnothing$. En efecto:

i. Sea la persona (1) conoce a la persona (i) y a la persona (i), además las personas (i) y (i) (por hipótesis) tienen como amigo común a la persona (D) entonces se cumple que $A_{i} \cap A_{j} \neq \varnothing$ pues (D) $\in A_{i} \cap A_{j}$. Si (1) conoce a (D) entonces las personas (1) y (D) tienen dos amigos comunes lo cual es una contradicción con la hipótesis.

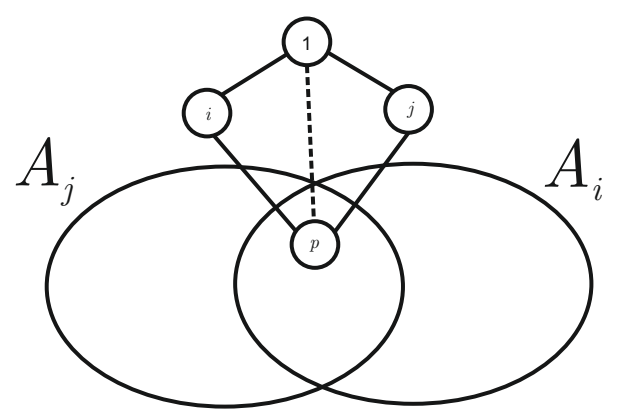

(i)

ii. Ahora, supongamos que la persona (1) conoce a la persona (D) y que el amigo común de (1) e (i) es (i) y además supongamos que (i) conoce a (D) pero (i) no conoce a (D). Entonces $A_{i} \cap A_{j} \neq \varnothing$, (1) e (i) tienen dos amigos comunes. Nuevamente llegamos a una contradicción con la hipótesis.

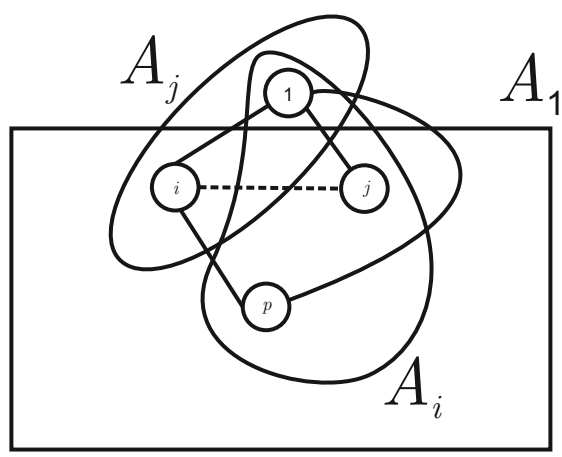

(ii)

Por lo anterior se tiene que (i) no debe conocer a (j), entonces $j \notin A_{i}$.

4) Continuamos con la demostración. Sea la persona (i) en la fiesta.

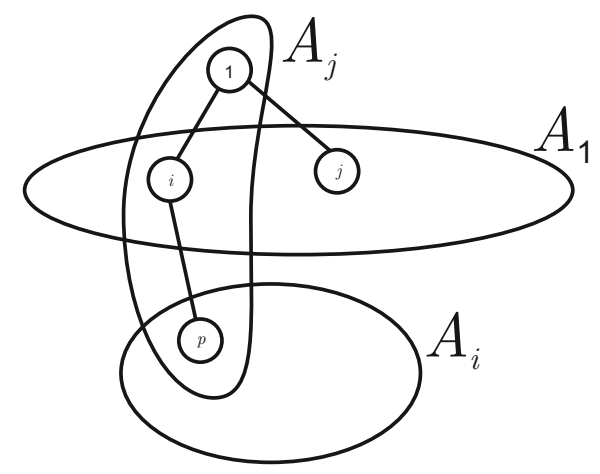


i. Si ( $(1)$ es amigo de (1) entonces $j \in A_{1}$ y $A_{1} \cap A_{i}=\varnothing$. Además $A_{j} \cap A_{i}=\varnothing$ pues $j \notin A_{i}$.

ii. Si (i) no es amigo de (1), entonces $j \notin A_{1}$ y por hipótesis existe un $i$ tal que $j \in A_{i}$ (cada dos personas tienen exactamente un amigo en común). En este caso estaríamos en el caso $i i$ de la parte 3) y se cumple que $A_{1} \cap A_{j}=\varnothing$ y por la parte $i$ de 4 ) $A_{i} \cap A_{j}=\varnothing$.

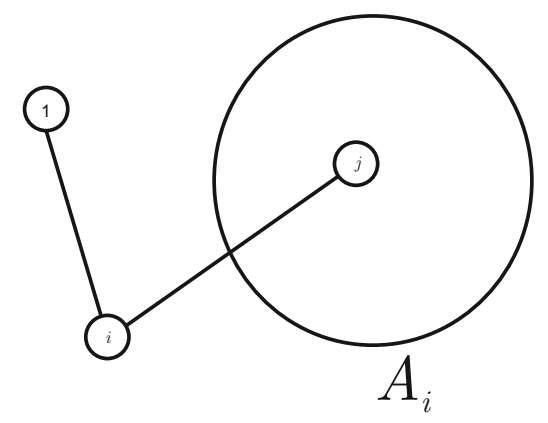

Asimismo, se cumple, que el conjunto total de personas en la fiesta está dado por (por ser $i$ amigo de 1 puede ser cualquiera de $2 \leqslant i \leqslant 2 k+1$ )

$$
\mathcal{F}=\{1\} \cup\left[\bigcup_{i=2}^{2 k+1} A_{i}\right]
$$

Esto prueba, que los $A_{i}$ son una partición de $\mathcal{F}$.

5) Por otro lado, probemos el paso 4: si alguien en la fiesta conoce exactamente solo a dos personas, entonces elegimos esta persona como (1) y por hipótesis existe un amigo en común.

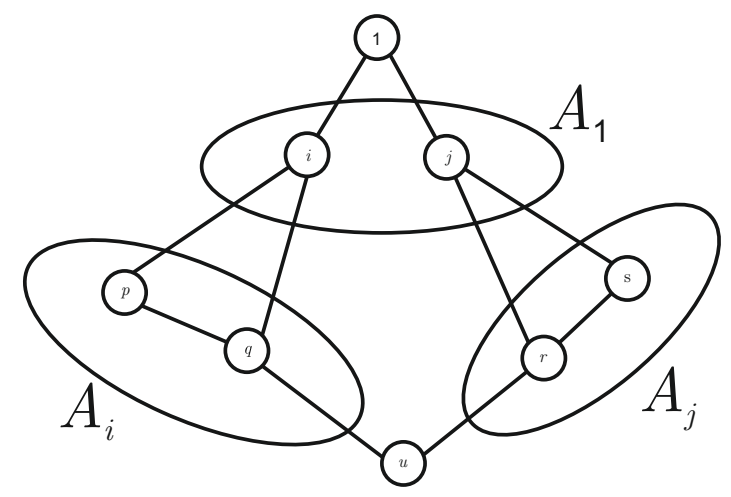

Por la parte 4) $\operatorname{los} A_{i}, A_{j}$ y $A_{1}$ satisfacen que $A_{i} \cap A_{1}=\varnothing, A_{i} \cap A_{j}=\varnothing$. Por otra parte,

a. Supongamos que $\left|A_{i}\right|=\left|A_{j}\right| \neq 0$. Entonces $\exists q \in A_{i}$ y $r \in A_{j}$.

i. Si $q$ y $r$ no son amigos entonces debe existir un nodo $u$ tal que sea el amigo común 


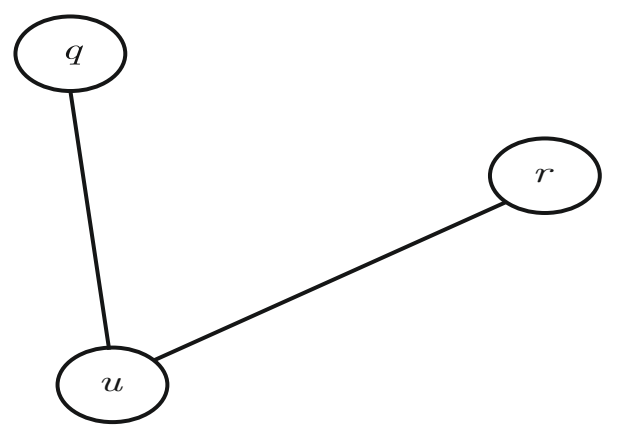

luego $A_{q} \cap A_{r} \neq \varnothing$, lo cual es una contradicción con la parte 4).

ii. Si $r$ y $q$ son amigos entonces $r, i \in A_{q}$, luego $A_{q} \cap A_{j} \neq \varnothing$ y $A_{q} \cap A_{1} \neq \varnothing$, lo cual es una contradicción.

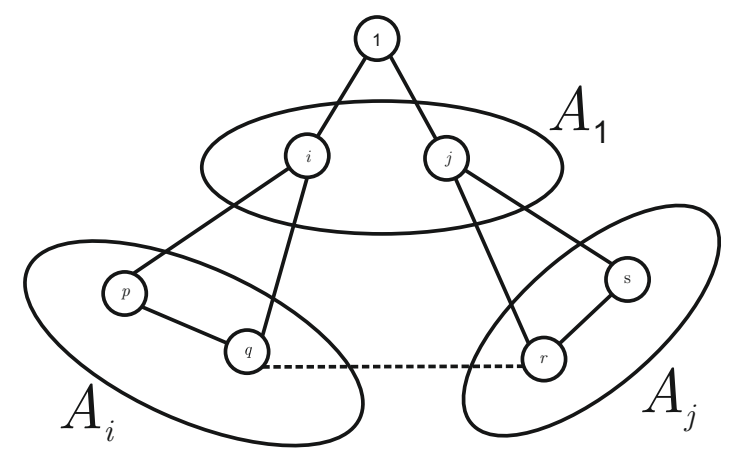

b. Supongamos ahora que $\left|A_{i}\right|>\left|A_{j}\right| \neq 0$. El esquema que usaremos será el siguiente:

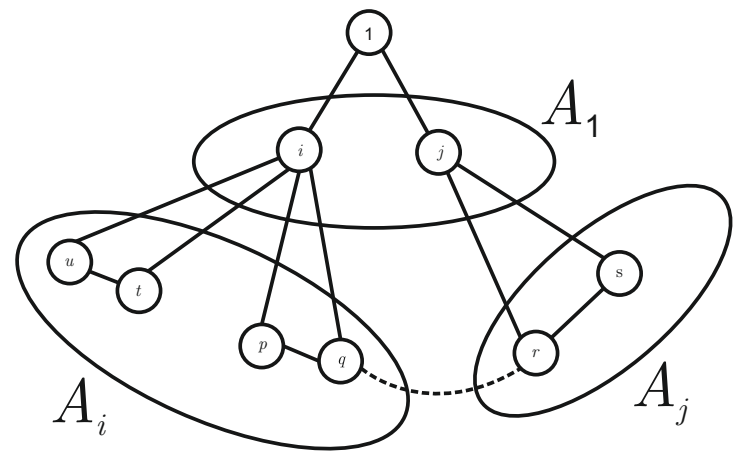

Para este caso, el análisis es el mismo que en el caso $a$.

i. Si $q$ y $r$ no son amigos entonces debe existir un $u$ tal que $r \in A_{u}$. Entonces $A_{q} \cap A_{r} \neq \varnothing$ y esto es una contradicción.

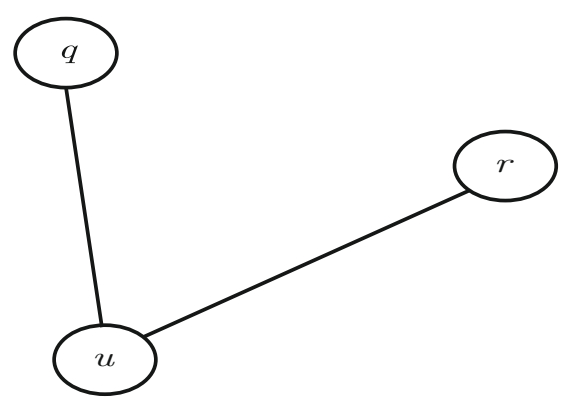


ii. Si $q$ y $r$ son amigos entonces $r, i \in A_{q}$ lo que implica que $A_{q} \cap A_{1} \neq \varnothing$ y $A_{q} \cap A_{1} \neq \varnothing$ lo cual es una contradicción.

Concluimos que en $\operatorname{los} \operatorname{casos} a$ ) y $b$ ) siempre se llega a una contradicción si $A_{j} \neq \varnothing$. Luego $A_{j}=\varnothing$ о $A_{i}=\varnothing$.

6) Ahora probemos que si la persona (1) conoce a más de dos personas (i) y (i), entonces: $\left|A_{i}\right|=$ $\left|A_{j}\right|$. En efecto, Supongamos que (i) y (i) son dos amigos de (1) que no se conocen. Entonces probaremos que $A_{i}$ y $A_{j}$ tienen el mismo número de elementos. Para ello, consideremos el siguiente esquema:

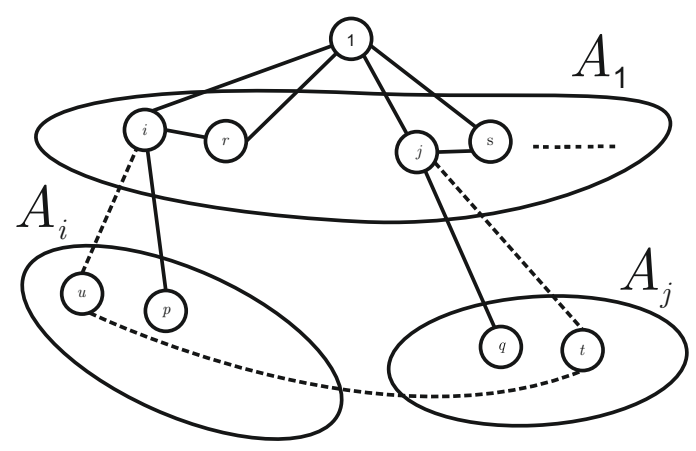

A. Si $A_{j} \neq \varnothing$ y $A_{i} \neq \varnothing$ entonces $q \in A_{j}, p \in A_{i}$.

i. En $A_{j}$ : Si $t \neq q$ entonces $\exists u \neq p, u \in A_{i}$. Pues de lo contrario, si $u=p$ implicaría $\overline{\text { que } p} \mathrm{y} j$ poseen dos amigos comunes. Por lo tanto el número de elementos de $A_{i}$ debe ser mayor o igual que el número de elementos de $A_{j}$

$$
\left|A_{i}\right| \geq\left|A_{j}\right|
$$

ii. En $A_{i}$ : Se procede en forma análoga y se prueba que

$$
\left|A_{i}\right| \leq\left|A_{j}\right|
$$

Por lo tanto $\left|A_{i}\right|=\left|A_{j}\right|$ cuando $i$ y $j$ no son amigos.

B. Si $A_{j}=\varnothing$ entonces $A_{i}=\varnothing$. En efecto, consideremos el siguiente esquema:

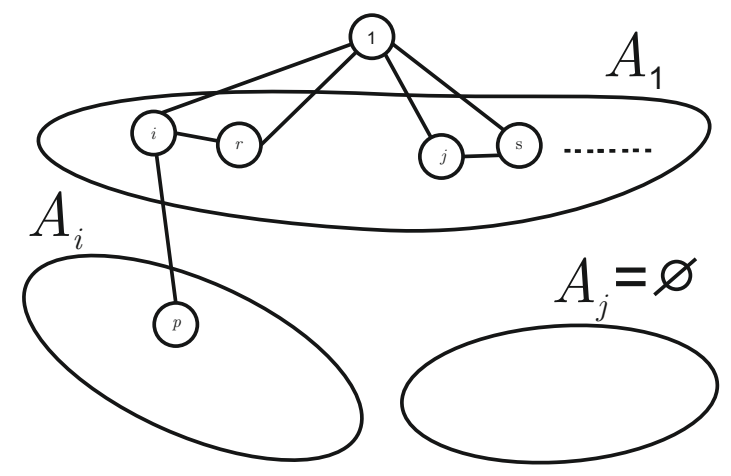

Supongamos que $A_{i} \neq \varnothing$, entonces $\exists p \in A_{i}$. Luego, el amigo común entre $p$ y $j$ que debe existir será (u) y $u \in A_{j}$ pero esto contradice el hecho de ser $A_{j}=\varnothing$. Por lo tanto no debe existir $p \in A_{i}$. Luego, $A_{i}=\varnothing$. 
7) Ahora probemos que todos $\operatorname{los} A_{i}(i=2,3, \ldots, 2 k+1)$ tienen el mismo número de elementos. En efecto, consideremos el siguiente esquema:

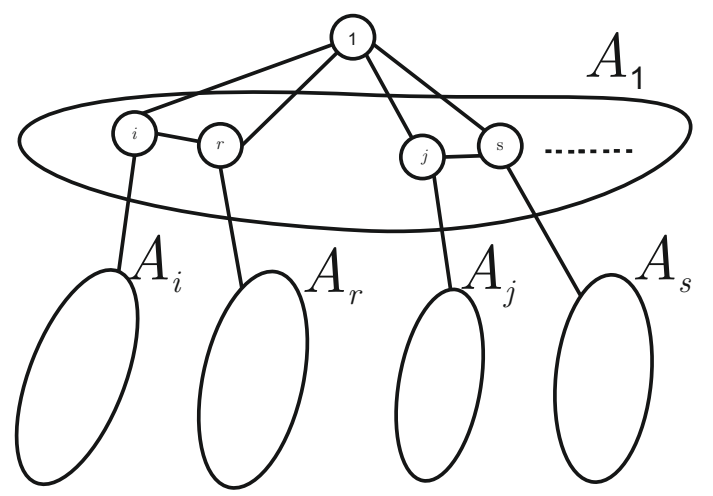

Como $i$ y $j$ no se conocen, entonces por la parte 6): $\left|A_{i}\right|=\left|A_{j}\right|$.

Como $i$ y $s$ no se conocen, entonces se tiene: $\left|A_{i}\right|=\left|A_{S}\right|$.

Como $r$ y $s$ no se conocen, entonces se tiene: $\left|A_{r}\right|=\left|A_{s}\right|$.

Como $r$ y $j$ no se conocen, entonces se tiene: $\left|A_{j}\right|=\left|A_{r}\right|$.

Luego: $\left|A_{i}\right|=\left|A_{j}\right|=\left|A_{r}\right|=\left|A_{s}\right|=\ldots$ y así sucesivamente.

8) Ahora, podemos concluir que, como (1) fue elegido en forma arbitraria, entonces el $r$ puede ser elegido como (1), entonces se cumple que

$$
\left|A_{1}\right|=\left|A_{j}\right|=\left|A_{s}\right|=\left|A_{i}\right|
$$

De aquí todas las personas en la fiesta tienen el mismo número de amigos.

9) Luego, como (1) tiene a (i) como amigo y $i=2,3, \ldots, 2 k+1$ entonces $A_{1}$ tiene $2 k$ elementos. Entonces para contabilizar el número total de personas en la fiesta utilizamos la fórmula (ii.)

$$
\begin{aligned}
& n=1+(2 k-1)(2 k) \\
& n=1+2 k(2 k)-2 k \\
& n=1+4 k^{2}-2 k
\end{aligned}
$$

10) Ahora, vamos a construir la matriz de adyacencia del grafo que representa la amistad entre las personas de la fiesta.

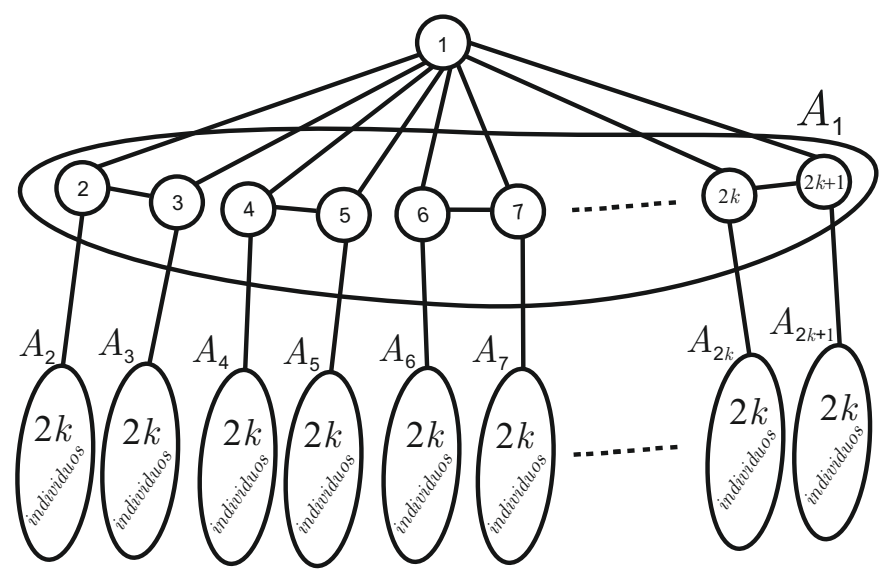


Usando la fórmula (ii.):

$$
\begin{aligned}
& n=1+\left|A_{1}\right|+\sum_{j=2}^{2 k+1}\left|A_{j}\right|=1+2 k+\sum_{j=2}^{2 k+1}\left|A_{j}\right| \\
& n=1+2 k+2 k \sum_{j=2}^{2 k+1} 1=1+2 k+2 k[2 k+1-2+1] \\
& n=4 k^{2}-2 k+1
\end{aligned}
$$

11) La matriz de adyacencia tendrá la siguiente forma:

$$
M=\left(\begin{array}{cccccccccc}
0 & 1 & 1 & 1 & 1 & 1 & \ldots & 1 & \cdots & 0 \\
1 & 0 & 0 & 1 & 1 & 0 & \ldots & 1 & \ldots & . \\
1 & 1 & 0 & 0 & 0 & 0 & \ldots & 1 & \ldots & 0 \\
1 & 1 & 0 & \ddots & \ldots & \ldots & \ldots & \ldots & \ldots & . \\
1 & 1 & 0 & \ldots & \ddots & \ldots & \ldots & \ldots & \ldots & . \\
1 & 0 & 1 & \ldots & \ldots & \ddots & \ldots & \ldots & \ldots & . \\
\vdots & \vdots & \vdots & \vdots & \vdots & \vdots & \ddots & \vdots & \vdots & \vdots \\
1 & . & . & \ldots & \ldots & \ldots & \ldots & \ddots & \ldots & . \\
\vdots & \vdots & \vdots & \vdots & \vdots & \vdots & \vdots & \vdots & \ddots & \vdots \\
0 & 1 & 0 & \ldots & \ldots & \ldots & \ldots & \ldots & \cdots & 0
\end{array}\right)_{n \times n}
$$

La matriz de adyacencia siempre es simétrica y cada fila o columna tendra $2 k$ entradas iguales a 1 (que refleja la cantidad de amigos de cada nodo).

12) Por otro lado debemos notar que la matriz $M$ tiene la siguiente propiedad:

La matriz $M^{2}$ va a reflejar el número de amigos comunes entre los nodos (o personas). Es decir la entrada $\left(M^{2}\right)_{i j}$ representa el número de amigos comunes entre las personas $i$ y $j$ y la entrada $\left(M^{2}\right)_{i i}$ representa el número de amigos que tiene la persona $i$.

13) La matriz $M^{2}$ tiene la siguiente forma:

$$
M^{2}=\left(\begin{array}{cccccccc}
2 k & 1 & 1 & 1 & 1 & \cdots & \cdots & 1 \\
1 & 2 k & 1 & 1 & 1 & \cdots & \cdots & 1 \\
1 & 1 & 2 k & 1 & 1 & \cdots & \cdots & 1 \\
1 & 1 & 1 & 2 k & 1 & \cdots & \cdots & 1 \\
1 & 1 & 1 & 1 & 2 k & \cdots & \cdots & 1 \\
\vdots & \vdots & \vdots & \vdots & \vdots & \ddots & \cdots & \vdots \\
\vdots & \vdots & \vdots & \vdots & \vdots & \cdots & \ddots & \vdots \\
1 & 1 & 1 & 1 & 1 & \cdots & \cdots & 2 k
\end{array}\right)_{n \times n}
$$

La cual puede ser escrita como

$$
M^{2}=U+(2 k-1) I
$$


donde

$$
U=\left(\begin{array}{cccccc}
1 & 1 & 1 & 1 & \cdots & 1 \\
1 & 1 & 1 & 1 & \cdots & 1 \\
1 & 1 & 1 & 1 & \cdots & 1 \\
1 & 1 & 1 & 1 & \cdots & 1 \\
\vdots & \vdots & \vdots & \vdots & \ddots & \vdots \\
1 & 1 & 1 & 1 & \cdots & 1
\end{array}\right), I=\left(\begin{array}{cccccc}
1 & 0 & 0 & 0 & \cdots & 0 \\
0 & 1 & 0 & 0 & \cdots & 0 \\
0 & 0 & 1 & 0 & \cdots & 0 \\
0 & 0 & 0 & 1 & \cdots & 0 \\
\vdots & \vdots & \vdots & \vdots & \ddots & \vdots \\
0 & 0 & 0 & 0 & \cdots & 1
\end{array}\right)
$$

14) Ahora, vamos a usar algunas propiedades del álgebra lineal para calcular el valor de $k$.

i. Lo primero que notamos es que las entradas de la matriz $M$ son puros unos (números enteros)

ii. Los autovalores de la matriz $M$, son las raíces cuadradas (positivas o negativas) de los autovalores de $M^{2}$.

iii. Es fácil verificar que $n$ es un autovalor para la matriz $U$ asociado al autovector $v=$ $(1,1,1, \ldots, 1)$

$$
U \cdot\left(\begin{array}{c}
1 \\
1 \\
\vdots \\
1
\end{array}\right)=\left(\begin{array}{c}
n \\
n \\
\vdots \\
n
\end{array}\right)=n \cdot\left(\begin{array}{c}
1 \\
1 \\
\vdots \\
1
\end{array}\right)
$$

iv. Por propiedad de traza de una matriz se cumple:

$$
n=\operatorname{traza}(U)=\sum_{i=1}^{n} u_{i}=n, \text { donde los } u_{i} \text { son los autovalores de } U
$$

Si $u_{1}=n$ entonces $\sum_{i=1}^{n} u_{i}=n+\sum_{i=2}^{n} u_{i}=n$. Luego $\sum_{i=2}^{n} u_{i}=0$.

15) Por otro lado, $\operatorname{traza}(M)=0$, entonces

$$
\operatorname{traza}(M)=\sum_{i=1}^{n} \lambda_{i}=0
$$

Así mismo

$$
\begin{aligned}
\operatorname{traza}\left(M^{2}\right) & =\operatorname{traza}(U)+\operatorname{traza}((2 k-1) I) \\
& =n+n(2 k-1)=n[1+2 k-1] \\
& =2 k \cdot n
\end{aligned}
$$

Por otro lado, para la matriz diagonal $(2 k-1) I=B$ se tiene que $p(\lambda)=\operatorname{det}(B-\lambda I)=0$, lo cual implica que $p(\lambda)=((2 k-1)-\lambda)^{n}$ es el polinomio característico entonces tiene como autovalor $\lambda_{i}^{\prime}=2 k-1, i=1,2, \ldots, n$ ( $n$ veces).

16) Por otro lado se cumple,

$$
\operatorname{det}|U-\lambda I|=\left|\begin{array}{ccccc}
1 & 1 & 1 & \cdots & 1 \\
1 & 1 & 1 & \cdots & 1 \\
1 & 1 & 1 & \cdots & 1 \\
\vdots & \vdots & \vdots & \ddots & \vdots \\
1 & 1 & 1 & \cdots & 1
\end{array}\right|-\lambda I=\left|\begin{array}{ccccc}
1-\lambda & 1 & 1 & \cdots & 1 \\
1 & 1-\lambda & 1 & \cdots & 1 \\
1 & 1 & 1-\lambda & \cdots & 1 \\
\vdots & \vdots & \vdots & \ddots & \vdots \\
1 & 1 & 1 & \cdots & 1-\lambda
\end{array}\right|
$$


Usando operaciones elementales fila, la $1^{\text {ra }}$ columna $C_{1}$ la multiplicamos por (-1) y sumamos a cada columna de la matriz $(U-\lambda I)$

$$
=\left|\begin{array}{ccccc}
1-\lambda & \lambda & \lambda & \cdots & \lambda \\
1 & -\lambda & 0 & \cdots & 0 \\
1 & 0 & -\lambda & \cdots & 0 \\
\vdots & \vdots & \vdots & \ddots & \vdots \\
1 & 0 & 0 & \cdots & -\lambda
\end{array}\right|=\left|\begin{array}{ccccc}
-\lambda & \lambda & \lambda & \cdots & \lambda \\
1 & -\lambda & 0 & \cdots & 0 \\
1 & 0 & -\lambda & \cdots & 0 \\
\vdots & \vdots & \vdots & \ddots & \vdots \\
1 & 0 & 0 & \cdots & -\lambda
\end{array}\right|+\left|\begin{array}{ccccc}
1 & \lambda & \lambda & \cdots & \lambda \\
0 & -\lambda & 0 & \cdots & 0 \\
0 & 0 & -\lambda & \cdots & 0 \\
\vdots & \vdots & \vdots & \ddots & \vdots \\
0 & 0 & 0 & \cdots & -\lambda
\end{array}\right|
$$

Ahora sumando la $2^{\text {da }}, 3^{\text {ra }}, 4^{\text {ta }}, \ldots, n$ filas a la $1^{\text {ra }}$ fila se obtiene

$$
=\left|\begin{array}{ccccc}
{[(n-1)-\lambda]} & 0 & 0 & \cdots & 0 \\
1 & -\lambda & 0 & \cdots & 0 \\
1 & 0 & -\lambda & \cdots & 0 \\
\vdots & \vdots & \vdots & \ddots & \vdots \\
1 & 0 & 0 & \cdots & -\lambda
\end{array}\right|+\left|\begin{array}{ccccc}
1 & \lambda & \lambda & \cdots & \lambda \\
0 & -\lambda & 0 & \cdots & 0 \\
0 & 0 & -\lambda & \cdots & 0 \\
\vdots & \vdots & \vdots & \ddots & \vdots \\
0 & 0 & 0 & \cdots & -\lambda
\end{array}\right|
$$

y por ser matrices triangulares

$$
\begin{aligned}
\operatorname{det}(U-\lambda I) & =[(n-1)-\lambda] \cdot(-\lambda)^{n-1}+(-\lambda)^{n-1} \\
& =(-\lambda)^{n-1} \cdot((n-1)-\lambda+1) \\
& =(-1)^{n-1} \cdot \lambda^{n-1} \cdot(n-\lambda)
\end{aligned}
$$

de donde $0=\operatorname{det}(U-\lambda I)=(-1)^{n-1} \cdot \lambda^{n-1} \cdot(n-\lambda)$ nos brinda los autovalores de $U, \lambda_{1}=$ $n \wedge \lambda_{i}=0, i=2,3, \ldots, n$ (autovalor cero de multiplicidad $n-1$ ).

Con esto queda también probada la afirmación en 13.

17) Por la parte 15$)$ se tiene que $B=(2 k-1) I$ es una matriz que tiene como autovalor a $\lambda_{1}^{\prime}=$ $(2 k-1)$ de multiplicidad $n$ y los autovalores de $U$ son $\lambda_{1}=n$ y $\lambda_{i}=0 i=2,3, \ldots, n-1$.

Por la forma de $M^{2}=U+(2 k-1) I$ un autovalor $\lambda_{1}$ de $U$ y $\lambda_{1}^{\prime}$ de $(2 k-1) I$ forman un autovalor de $M^{2}$.

En efecto sea $\theta$ autovalor de $M^{2}$ entonces

$$
M^{2} v=\theta v
$$

lo que implica que

$$
(U+(2 k-1) I) v=\theta v
$$

por lo tanto

$$
U v+(2 k-1) I v=\theta v
$$

y tenemos que

$$
\begin{aligned}
\lambda_{1} v+\lambda_{1}^{\prime} v & =\theta v \\
\left(\lambda_{1}+\lambda_{1}^{\prime}\right) & =\theta v
\end{aligned}
$$

Luego:

$$
\begin{aligned}
& \theta=\lambda_{1}+\lambda_{1}^{\prime} \\
& \theta=n+2 k-1 .
\end{aligned}
$$


Entonces los autovalores de $M^{2}$ pueden ser formados

$$
\begin{array}{rll}
\theta_{1} & =\lambda_{1}+\lambda_{1}^{\prime}=n+2 k-1 \\
\theta_{2} & =\lambda_{2}+\lambda_{2}^{\prime} & =0+2 k-1=2 k-1 \\
\vdots & \vdots & \vdots \\
\theta_{n} & =\lambda_{n}+\lambda_{n}^{\prime} & =0+2 k-1=2 k-1
\end{array}
$$

Luego, los autovalores de $M$ deben ser las raíces cuadradas (positivas o negativas) de los autovalores de $M^{2}$

$$
\sigma_{1}=\sqrt{n+2 k-1} \text { у } \sigma_{i}=\sqrt{2 k-1} i=2,3, \ldots, n
$$

Entonces, por la traza de $M$ igual a cero, se tiene

$$
0=\operatorname{traza}(M)=\sum_{i=1}^{n} \sigma_{i}=\sqrt{n+2 k-1}+\sqrt{2 k-1}+\ldots+\sqrt{2 k-1}
$$

entonces existe $a \in \mathbb{Z}$ tal que

$$
\sqrt{n+2 k-1}=a \sqrt{2 k-1}
$$

Por otro lado recordemos que $k$ es un entero positivo, luego

$$
n+2 k-1=a^{2}(2 k-1)
$$

y por la relación (2) se tiene

$$
4 k^{2}=a^{2}(2 k-1)
$$

De donde la única solución resulta para $k=1$. Luego, usando la relación $n=4 k^{2}-2 k+1$ se concluye que $n=3$. Con lo cual queda probado el teorema.

\section{Conclusiones}

El uso de la teoría de grafos y del algebra lineal nos ha permitido brindar otra demostración de una variante del famoso teorema de la amistad de Ramsey. De esta manera se muestra que se pueden llegar a los mismos resultados siguiendo caminos diferentes. 


\section{Referencias Bibliográficas}

[1] Erdos, P., Rényi, A., Sós, V. (1966). On a problem of graph theory Hungar. Studia Sci. Math.1: 215-235.

[2] Ramsey, F. (1930) On a problem of Formal Logic. Proc. London Math. Soc. S2-30 (1): 264-286.

[3] Chvátal, V., Kotzig, A., Rosenberg, I., Davies, R. (1976). There are $2^{N_{\alpha}}$ friendship graphs of cardinal $N_{\alpha}$. Canadian Mathematical Bulletin 19 (4): 431-433.

[4] Foulds, L. (1992). Graph Theory Applications. New York, Springer Verlag.

[5] Lang, S. (1987). Linear Algebra. Third Edition, New York, Springer Verlag. 